\section{Trotz Corona-} Shutdown: keine Entwarnung beim Klimaschutz

Die Corona-Krise hat das Thema Klimaschutz derzeit aus den Schlagzeilen verdrängt. Dabei wäre es gerade jetzt geboten, die notwendigen Schlüsse aus den Entwicklungen der letzten Monate zu ziehen. Denn die in der ersten Hälfte des Jahres nahezu auf der gesamten Welt verhängten Maßnahmen, um die Ausbreitung der Covid-19-Pandemie zu begrenzen, hatten auch wesentliche Auswirkungen auf den Kohlendioxid-Ausstoß. Wie die Fachzeitschrift „Nature Climate Change“ jüngst berichtete, gingen die weltweiten $\mathrm{CO}_{2}$-Emissionen in den vergangenen Monaten zeitweise um etwa ein Sechstel zurück. Die globalen Tageswerte waren Anfang April um bis zu $17 \%$ niedriger als im Durchschnitt des Jahres 2019. Nach Angaben der englischen University of East Anglia konnte der 7. April 2020 als der Tag mit dem bislang weltweit größten $\mathrm{CO}_{2}$-Rückgang identifiziert werden. An diesem Tag sind schätzungsweise 17 Millionen Tonnen Kohlendioxid weniger durch die Verbrennung fossiler Energieträger erzeugt worden als an einem durchschnittlichen Tag im vergangenen Jahr.

Diese Zahlen sind aber leider kein Grund für Jubelmeldungen. Und schon gar keiner für eine Entwarnung. Zum einen werden nämlich trotz des Lockdowns neue $\mathrm{CO}_{2}$-Rekorde gemessen: Im Mai wurde ein Konzentrationswert von rund 418 ppm festgestellt, 2019 war der Höchstwert noch bei $415 \mathrm{ppm}$ gelegen. Damit ist der $\mathrm{CO}_{2}$-Gehalt in der Atmosphäre seit der vorindustriellen Zeit um rund $50 \%$ angestiegen. Zum anderen wird das Ausmaß der auch zukünftig noch notwenigen Klimaschutzmaßnahmen deutlich gemacht - und dieses ist beachtlich: Um die Erderwärmung wie im Pariser Abkommen vorgesehen auf 1,5 Grad Celsius im Vergleich zum vorindustriellen Zeitalter zu begrenzen, müssten die Emissionen nicht nur einmalig wie im Jahr 2020, sondern jedes Jahr wieder um jeweils acht Prozent sinken. Nur dann ist das Ziel der Klimaneutralität bis zur Mitte des Jahrhunderts zu erreichen. Der Corona-Lockdown hat uns gezeigt, wie groß diese Aufgabe eigentlich ist, vor der wir nach wie vor stehen.

Daniel Ennöckl

\section{Judikatur-Highlights}

\section{(VERFASSUNGSKON- FORMES) PLASTIKSA- CKERL-VERBOT}

Der VfGH (17.06.2020, G 227/2019) hat den (Individual-)Antrag auf Aufhebung mehrerer Bestimmungen des AWG 2002 abgelehnt:

Im Zuge der AWG-Rechtsbereinigungsnovelle 2019 (BGBl I 71/2019) wurden u.a. Bestimmungen eingefügt, um negative Auswirkungen von Kunststofftragetaschen auf die Umwelt, insbesondere das Gewässer und die menschliche Gesundheit zu verhindern und die Kreislaufwirtschaft zu fördern (IA 887 26. GP 9). Eine wesentliche Maßnahme zur Reduktion von Plastikabfällen in Österreich ist ein generelles Verbot von Kunststofftragetaschen ab dem 1. Jänner 2020 (mit nur vereinzelten Ausnahmen wie zB biologisch abbaubare und aus nachwachsenden Rohstoffen hergestellte Knotenbeutel). Die Antragsteller erachteten das Verbot als verfassungswidrig.

Das Verbot des Inverkehrsetzens von Kunststofftragetaschen liegt im gesetzgeberischen Gestaltungsspielraum, ist hinreichend bestimmt und der Eingriff in das Recht auf Freiheit der Erwerbsbetätigung ist verhältnismäßig: Dabei bezieht sich der VfGH auf unionsrechtliche Vorgaben und die hohe Bedeutung des Umweltschutzes (mit Verweis auf Rechtsprechung zum Binnenschifffahrts-KonzessionsG und zum LuftFG iZm der dritten Piste für den Flughafen Wien-Schwechat). Gewisse umweltschonende Tragetaschen sind vom Verbot ausgenommen; diese Ausnahmen sind auch hinreichend bestimmt. Verfassungsrechtlich ist es auch nicht geboten, Produkte $\mathrm{zu}$ dem Zweck herzustellen, dass diese in weiterer Folge dem Recyclingkreislauf zugeführt werden können. Der Eingriff in die Erwerbsfreiheit ist insofern verhältnismäßig, als die gesundheits- und umweltpolitische Zielsetzung die Schwere des Eingriffs in die Rechte von Kunststofftragetaschen-Herstellern überwiegt.

\section{RECHTSWIDRIGE INTER- ESSENABWÄGUNG}

Das LVwG Tirol gab einer Beschwerde des Tiroler Landesumweltanwaltes statt und versagte damit der antragstellenden Partei die naturschutzrechtliche Bewilligung zur Errichtung einer Sommer- und Winterrodelbahn (TNSchG). Durch das Projekt würde in ein Feuchtgebiet eingegriffen sowie geschützte Lebensräume und geschützte Pflanzen- und Tierarten erheblich beein- trächtigt. Die Projektalternative Seilgarten wäre wesentlich weniger eingriffsintensiv. Den Auswirkungen auf die Schutzgüter „Feuchtgebiet, Vogelarten und Landschaftsbild" sei die Förderung des Tourismus gegenüber zu stellen. Letzteres komme nicht in Betracht, wenn weniger beeinträchtigende Alternativen zur Verfügung stehen.

In der Revision wird die vorgenommene Interessenabwägung sowie das Vorliegen einer weniger eingriffsintensiven Alternative (Seilgarten) beanstandet.

Der VwGH hob die Entscheidung des LVwG auf (VwGH 09.06.2020, Ra 2019/10/0075): Das VwG hat hinsichtlich des Überwiegens der langfristigen öffentlichen Interessen an der Bewilligungserteilung über die Naturschutzinteressen eine Wertentscheidung zu treffen. Hier lag keine transparente und nachvollziehbare Wertentscheidung vor: Es fehlten bereits hinreichende Feststellungen zu den beeinträchtigten Naturschutzinteressen sowie zu den langfristigen öffentlichen Interessen an der beantragten Rodelbahn.

Zudem hat sich das LVwG nicht damit auseinandergesetzt, ob die Projektalternative Seilgarten die gesetzlichen Anforderungen erfüllt und zwar, ob der angestrebte Zweck mit einem im Verhältnis zum erzielbaren Erfolg vertretbaren Aufwand auf eine andere Weise erreicht werden kann.

Auch das Fehlen der Voraussetzungen für eine Ausnahme, was zur Versagung der Bewilligung führt, bedarf einer ausreichenden rechtlichen Auseinandersetzung mit dem Projekt. Die Ausführungen, dass bestimmte durch die Tiroler Naturschutzverordnung 2006 geschützte Lebensräume durch das Projekt „berührt“ würden bzw. geschützte Pflanzen im Projektgebiet „vorgefunden“ worden seien, reicht nicht.

\section{AUSLEGUNG DER UNMIT- TELBAREN BETROFFEN- HEIT}

GA Bobek(SAvom 16.07.2020, Rs. C-352/19P) plädiert dafür, eine regionale oder lokale Gebietskörperschaft, wie die Region BrüsselHauptstadt, als - sowohl unmittelbar als auch individuell - von einem Unionsrechtsakt betroffen zu erachten. Er bejaht damit die Klagebefugnis, hier betreffend die Durchführungsverordnung (EU) 2017/23241 der Kommission zur Erneuerung der Genehmigung des Wirkstoffs Glyphosat, weil die Region den Wirkstoff für gefährlich hält und die Verwendung in ihrem Gebiet untersagt.

Chiara Rockenschaub 


\section{News von den Gesetzgebern}

\section{NOVELLE DER DEPONIE- VERORDNUNG 2008}

Die zuletzt 2016 geänderte Deponieverordnung 2008 (DVO 2008) wird novelliert. Das Bundesministerium für Klimaschutz, Umwelt, Energie, Mobilität, Innovation und Technologie hat den entsprechenden Novellenentwurf zur Begutachtung ausgesendet; die Begutachtungsfrist endete am 7. September 2020.

Die Novelle verfolgt im Wesentlichen drei Regelungsschwerpunkte:

1. Umsetzung von Unionsrecht: Im Rahmen des im Juni 2018 veröffentlichten Kreislaufwirtschaftspakets der Europäischen Union wurde auch die EU-Richtlinie über Abfalldeponien geändert. Mit gegenständlicher Novelle sollen diese Änderungen nun in die DVO 2008 integriert werden. Insbesondere wird in Umsetzung der europarechtlichen Vorgaben ein Deponieverbot für Abfälle normiert, die für die Vorbereitung zur Wiederverwendung oder für das Recycling getrennt gesammelt werden, für bestimmte Abfallarten, die gemäß Recycling-Baustoffverordnung getrennt $\mathrm{zu}$ sammeln sind sowie für Gipsabfälle (Gipsplatten Gips-Wandbauplatten etc.)
2. Ablagerung von künstlichen Mineralwollabfällen: Um die ordnungsgemäße Abfallbeseitigung künstlicher Mineralwollabfälle mit gefahrenrelevanten Fasereigenschaften sicherzustellen, werden für diese neue, an Asbestabfälle angelehnte, Vorgaben aufgenommen. Zur Forcierung des Recyclings soll die Ablagerungsmöglichkeit auf sieben Jahre beschränkt werden.

3. Notfalllager für Abfälle im Katastrophenfall: Durch die Novelle wird die Möglichkeit geschaffen, Siedlungsabfälle, die in einem Katastrophenfall keiner ordnungsgemäßen Abfallbehandlung zugeführt werden können, in speziell dafür eingerichteten und den Vorgaben der DVO 2008 entsprechenden Notfalllagern zwischenzulagern.

\section{UMWELTFÖRDERUNG}

Im Rahmen des von der Bundesregierung vereinbarten Konjunkturpakets, welches die Konjunktur nach den durch die COVID-19-Krise verursachten Einschnitten wieder ankurbeln soll, werden auch für die Umweltförderung im Inland umfangreiche Mittel zur Verfügung gestellt. Vor diesem Hintergrund wird durch eine Novelle des Umweltförderungsgesetzes (UFG-Novelle 2020 - Konjunkturpaket) insbesondere die Fortführung der Anreizförderung für Investitionen zum Ersatz fossiler durch kli- mafreundlicher Heizungssysteme sowie der Förderung betrieblicher und kommunaler Umwelt- und Klimaschutzinvestitionen in den Jahren 2021 und 2022 sichergestellt. Die Novelle steht, nach Durchführung eines Begutachtungsverfahrens, kurz vor ihrer Erlassung.

Reka Krasznai

Hinweis des Verlags Der Verlag bleibt in Hinblick auf geografische Zuordnungen und Gebietsbezeichnungen in veröffentlichten Karten und Institutsadressen neutral.

\section{Praxistipp}

\section{MAKRO- UND MIKROPLASTIK}

Grundsätzlich wird zwischen primären und sekundärem Mikroplastik unterschieden; primäre Mikropartikel werden gezielt in gewünschter Größe insbesondere für die Kosmetikindustrie hergestellt, wie z. B. für Duschgels, Handwaschseifen, Zahnpasta, Shampoos oder Peelings, wo sie als „Schleifmittel" verwendet werden.

Sekundäre Mikropartikel entstehen unbeabsichtigt durch Alterungs- und Zerfallsprozesse von in die Umwelt eingetragenen Makroplastikteilen („Littering“).

Eine der wichtigsten Quellen von Mikroplastik ist Kleidung aus synthetischen Textilfasern, wie z. B. Polyester, Polyamid, Elastan oder Acryl. Dieses verliert bei jedem Waschvorgang hunderttausende Mikroplastikfasern, welche weder durch Waschmaschinen noch durch Kläranlagen aus dem Wasser gefiltert werden können.

In den Weltmeeren wird deshalb ein zunehmendes Auftreten von Mikroplastik be- obachtet. Zwar beträgt der Eintrag an Mikroplastik nur 0,1 bis $1,5 \%$ des gesamten Plastikeintrags, jedoch kann es dadurch zu einer Verfrachtung in die menschliche Nahrungskette durch Fisch und Meeresfrüchte kommen. Letztere verwechseln Mikroplastik mit Plankton und nehmen dieses als Nahrung auf.

Für den einzelnen Konsumenten ist die Verwendung zertifizierter Naturkosmetik eine gute Alternative zur Vermeidung von Mikroplastik. Ebenso die Verwendung von Kleidung aus Naturfasern wie Baumwolle, Leinen, Hanf, Wolle, Seide, etc. Jeder Verbraucher kann außerdem durch die richtige Entsorgung seiner kunststoffhaltigen Abfälle („Makroplastik“) einen wesentlichen Beitrag zum Kunststoffrecycling leisten.

Aus umweltpolitischer Sicht ist die Umsetzung des Verbots von Einwegkunststoffartikel zwingend geboten. Ebenso sind Maßnahmen zur Erhöhung des Anteils wiederverwendbarer Mehrwegflaschen sowie Bepfandung von Einweg-Kunststoffverpackungen vorzusehen.

Peter Hodecek

\section{Impressum/Beirat}

Univ.-Prof. Dr. Iris EISENBERGER, M.Sc. (LSE), Karl-Franzens-Universität Graz

Prof. Dr. Daniel ENNÖCKL, LL.M., Universität Wien MR Mag. Christian JANITSCH, MBA, BMK Mag. Martin NIEDERHUBER, Niederhuber \& Partner Rechtsanwälte

HR Mag. Dr. Edwin RADER

Dr. Peter SANDER, LL.M./MBA, Niederhuber \& Partne Rechtsanwälte

Univ.-Prof. MMag. Dr. Eva SCHULEV-STEINDL, LL.M. Karl-Franzens-Universität Graz

Dr. Florian STANGL, LL.M., Niederhuber \& Partner Rechtsanwälte

MMag. David SUCHANEK, Niederhuber \& Partner Rechtsanwälte

Redaktion: Mag. Fritz Randl, Österreichischer Wasser- und Abfallwirtschaftsverband (ÖWAV) randl@oewav.at

Mag. Heidrun Schiesterl, MA, Österreichischer Wasser-und Abfallwirtschaftsverband (ÖWAV), schiesterl@oewav.at

Zitiervorschlag: ÖWAW URK 2020, Seite\# 